\title{
Control and postural thixotropy of the forearm muscles: changes caused by cold
}

\author{
M LAKIE, EG WALSH, GW WRIGHT \\ From the Department of Physiology, University Medical School, Edinburgh, Scotland
}

SUMMARY The forearm was cooled in water at $5-10^{\circ} \mathrm{C}$ while wrist biodynamics were investigated. Pronounced loosening following a perturbation (thixotropy) was no longer seen. The wrist became stiffer for large or moderate but not small movements; EMG activity did not increase. Cooling the wrist alone, or opposite forearm, was without effect. The ability to make rapid reciprocating movements was reduced and muscle relaxation time was increased. Single movements were not affected.

It has long been a belief of physiologists that cold increases muscle tone, and people who are cold often experience a sensation of stiffness. In the classical description of a victim of hypothermia (rectal temperature $18^{\circ} \mathrm{C}$ ), Laufman ${ }^{1}$ observed that the neck muscles were rigid and the elbows could be flexed only with great force. The influence of temperature on muscle contraction and energy metabolism has been extensively investigated ${ }^{23}$ but there appears to be less information about the degree to which cooling changes the properties of relaxed muscles and about the mechanisms which underlie these changes. Cold also has severe deleterious effects on manual performance. ${ }^{4}$

Muscle tone, the resistance of the limbs to passive movement, is often assessed by manipulation, but a more accurate and revealing investigation is to subject the limb to mechanically produced forces. By using a torque motor to oscillate the wrist the stiffness and damping of the forearm muscles can be estimated. ${ }^{5}$ Postural thixotropy, where the motion produced by a rhythmic torque depends on the history of movement, can be revealed in this way. We have now investigated the behaviour of the wrist when the forearm is cooled.

\section{Methods}

The forearm was placed in a metal tank; the wrist and hand protruded through a flanged opening at one end (fig 1). A water-tight seal was provided by a surgical rubber glove with the fingers removed; the cut end of the glove was everted

Address for reprint requests: Dr EG Walsh, Department of Physiology, University Medical School, Teviot Place, Edinburgh EH8 9AG, UK.

Received 5 October 1984 and in revised form 13 March 1985. Accepted 23 March 1985 and fixed over the flange. Water was pumped continuously through the tank from reservoirs at a known temperature; the forearm was completely submerged. The wrist was concentric with the axis of rotation of the torque motor which produced flexion/extension movements of the joint in the horizontal plane. The motor was attached to the hand by a light crank and Velcro strapping. A G6M4 printed motor (Printed Motors Ltd., Borden, Hants), was modified to reduce friction in a manner similar to that described by Marsden et al; 6 it was fitted with a concentric low friction/low inertia precision plastic potentiometer to record the movement. Some experiments employed a very low friction basket wound motor (Philips MO10) with an integral tachometer which signalled angular velocity. The motors were driven by a class B amplifier which employed current feed-back to ensure that the output current (and thus the torque from the motor) was a faithful reproduction of the input wave form. The necessary waveforms were generated by an analogue circuit. The applied torque (from the current signal), the displacement (from the potentiometer) and the velocity (from the tachometer, or obtained by electronic

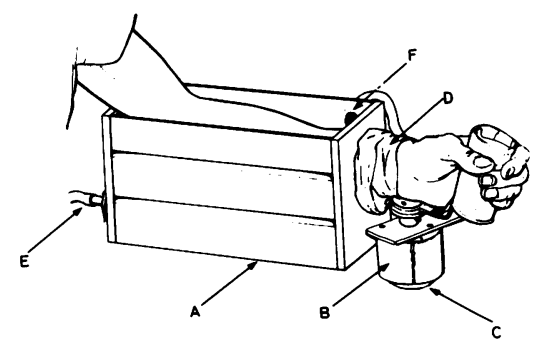

Fig 1 Apparatus. The arrangement of the cooling water bath $(A)$, the wrist seal (D), and printed motor $(B)$ is shown. The potentiometer $(C)$ is beneath the motor. Water enters at $(E)$ and exits at $(F)$. The hand is secured to the handle with light Velcro strapping. The Philips motor is similar, but it has an integral tachometer and is not fitted with a potentiometer. 

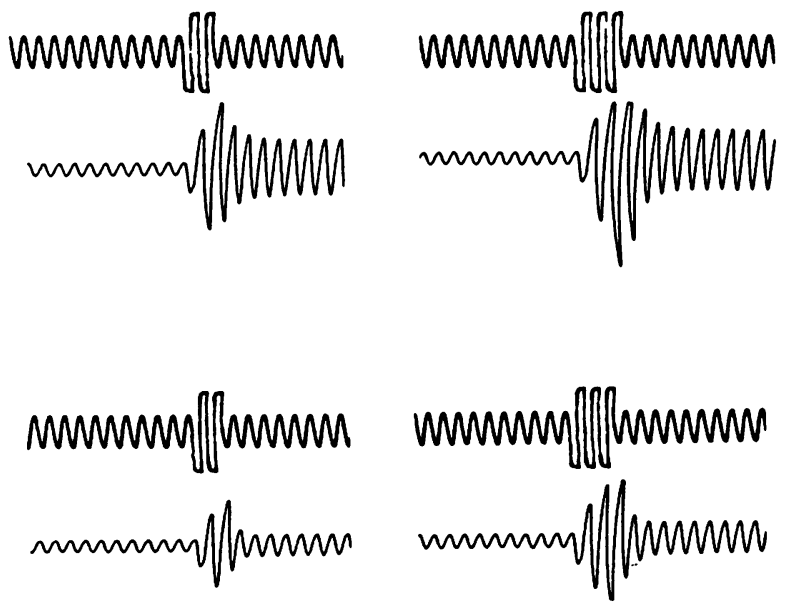
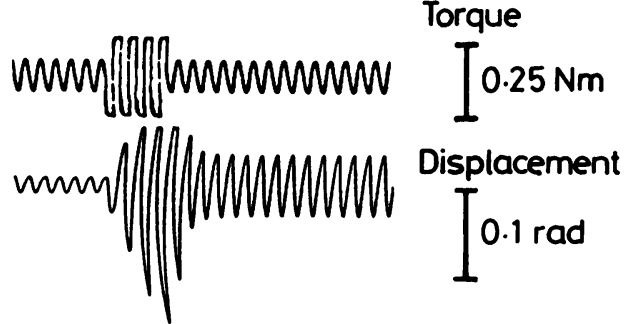

Displacement T $0.1 \mathrm{rad}$

Torque
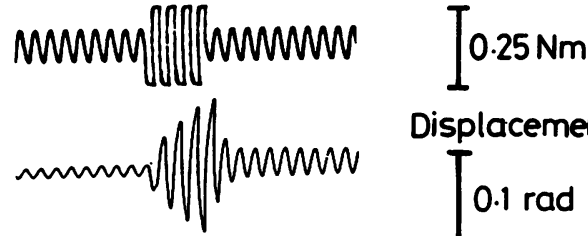

Displacement To.1 rad

\section{is}

Fig 2 Thixotropy. The (upper) control records show the large increase in movement that follows a short lasting larger perturbation of 2, 3 and 4 cycles respectively. The increase is the same in each case; the stiffness has been decreased by prior movements. Cooling (40 min at $8^{\circ} \mathrm{C}$, lower records) reduces the degree of loosening that follows a perturbation but does not change the stiffness that obtains before the perturbation. The size of the movement caused by the perturbation is much less following cooling.

differentiation of the position signal) were recorded on a multichannel rectilinear inkwriter. For the experiments on thixotropy a bistable was used to control electronic gates so that one or more cycles of the sinusoidal torque could be replaced by a larger rectangular force. The circuitry, calibrating and recording system have been previously described. 5

Other experiments were performed using a "hanging hand" tremorograph. ${ }^{78}$ The hand was slung from an induction generator supplied with direct current on one of its two windings. This instrument was almost frictionless and had negligible inertia; the non-energised winding provided a signal corresponding to acceleration. ${ }^{8}$

For the tracking experiments the hand was coupled to an identical induction generator and a concentric potentiometer in tandem. Position and acceleration could be recorded simultaneously, and the movement, which was extension/flexion in the horizontal plane, was loaded to a negligible extent. In these experiments a waveform generator controlled one spot on a large screen oscilloscope. A second spot was controlled by movements of the subject's wrist, the person being instructed to keep his spot aligned with the other as it moved horizontally. The position of the two spots was recorded on an inkwriter. The error signal was computed by an operational amplifier and the zero was inserted by multiplexing at $22 \mathrm{~Hz}$. The resulting trace was a blackened area with a height proportional to the mismatch between the two spots.

Surface recordings of EMG were made in most of the experiments. Suction cup EMG electrodes placed over the forearm flexor and extensor muscles worked statisfactorily despite immersion. Intramuscular fine wires and needle electrodes were occasionally used.
In experiments involving electrical stimulation of the forearm muscles stimuli (duration $100 \mu \mathrm{s}$ ) at up to 400 volts were applied to the motor point of extensor digitorum communiso using suction cup electrodes. A Devices clinical stimulator (Type 3072) was used.

All measurements of temperature of the skin and watero were made with a multi-channel telethermometer (YellowSprings Instrument Corp) and small surface thermistofo probes. The room temperature was warm, in the range $25-30^{\circ} \mathrm{C}$. Control observations were made with water at $35^{\circ} \mathrm{C}$ which was the approximate temperature of the skin of the forearm measured at the elbow; subjectively it was neither warm nor cold. There was no difference between measurements in air and in water at this temperature. The cold water was normally in the range $5-10^{\circ} \mathrm{C}$. Under these conditions the experiments were not uncomfortable and the initial sense of chill faded rapidly. More discomfort was caused by cooling a hand in water at a similar temperature; movements of abduction and adduction of the fingers were then affected much more than those of flexion and extension which depend primarily on the muscles of the forearm rather than the intrinsic muscles of the hand. There was never any shivering or general feeling or cold, but the immersed skin developed gooseflesh and redness sharply demarcated at the water level. The skin temperature of the hand was measured and it was about $5^{\circ} \mathrm{C}$ cooler than the control side. To prevent this the hand was sometimes warmed by an infra-red lamp or insulated by a glove. There was no obvious numbness, diminution of touch sensitivity or loss of proprioceptive sense in the fingers suggesting that the conduction of the sensory neurones passing through the cooled forearm was not seriously disturbed. At times temperatures of $1-3^{\circ} \mathrm{C}$ were used but this inevitably caused some persistent discomfort. 
Some observations were made during rewarming; effects that took over 30 minutes to appear in cold water disappeared after only 10 minutes rewarming $\left(35^{\circ} \mathrm{C}\right)$. On occasion measurements were made at one wrist as the other was cooled; no changes were observed. The subjects were volunteers of both sexes; information about the number, age and sex of the subjects is contained in the results section. The informed consent of each subject was obtained.

\section{Results}

\section{Changes in thixotropy and stiffness}

Thirteen experiments were performed on eight subjects, six were male. The mean age of the male subjects was 33, and of the females, 23. In this experiment, as in the ones described later, similar changes were observed in each subject.

Under certain conditions the motion of a limb resulting from rhythmic sinusoidal torques of low frequency (for example 1-3.5 Hz) depends strikingly on the past history of movement. We have called this property postural thixotropy. ${ }^{5}$ Thus, if the wrist is driven by a torque appropriate in frequency (for example $3 \mathrm{~Hz}$ ) and amplitude and a transient perturbation is applied, a large and self-perpetuating increase in the amplitude of movement occurs. The perturbation can be applied by the driving motor, but a voluntary movement of the wrist is equally effective. If the motion is arrested for about $2 \mathrm{~s}$ and the rhythmic force reapplied the resulting amplitude of movement will again be small. ${ }^{5}$ The amplitude of movement when the small rhythmic torque was first applied was not altered by cooling, but a perturbation, even if large or repeated, had a much reduced effect. The reduction became noticeable after about $15 \mathrm{~min}$ in water at $5^{\circ} \mathrm{C}$, and after $40 \mathrm{~min}$ the effect was strikingly decreased (fig 2). Even if many active or passive movements were made the system would not fully "loosen itself up". As the thixotropic effect is present in anaesthetised and paralysed patients at normal temperatures ${ }^{5}$ it cannot be neurally determined and the failure to loosen up following cooling must presumably be due to a direct effect on the muscle itself. During these observations the EMG was silent.

In 18 experiments (four male subjects mean age 22 $\mathrm{yr}, 12$ female subjects, mean age $21 \mathrm{yr}$ ) the motor applied low frequency (for example $1 \mathrm{~Hz}$ ) alternating torques to the hand pushing it into flexion and extension in turn. The torque was modulated so that successive cycles became gradually larger, attained a maximum, and then the programme repeated. In this way the stiffness of the limb could be monitored as the movement increased. At normal temperatures it was always found that the relaxed wrist was disproportionately stiff for small movements but became more compliant as the size of the movement increased and eventually started to become stiffer again with the largest forces as the anatomical limits of the joint were approached (fig 3A). The torque and the resulting displacement could be recorded on magnetic tape and then played back at lower speed onto a X-Y plotter. By jointing up the points where the system was stationary a composite compliance curve for the wrist could be obtained. In fig 3B the data from fig $3 A$ has been plotted in this way. The stationary points have not been jointed together to permit visualisation of how the curve is constructed. The three regions: initially very stiff, then compliant and finally becoming stiffer again are apparent. It was always found that the compliance was much less below a certain critical torque size. This corresponded to the increase in stiffness that is seen when small sinusoidal torques are

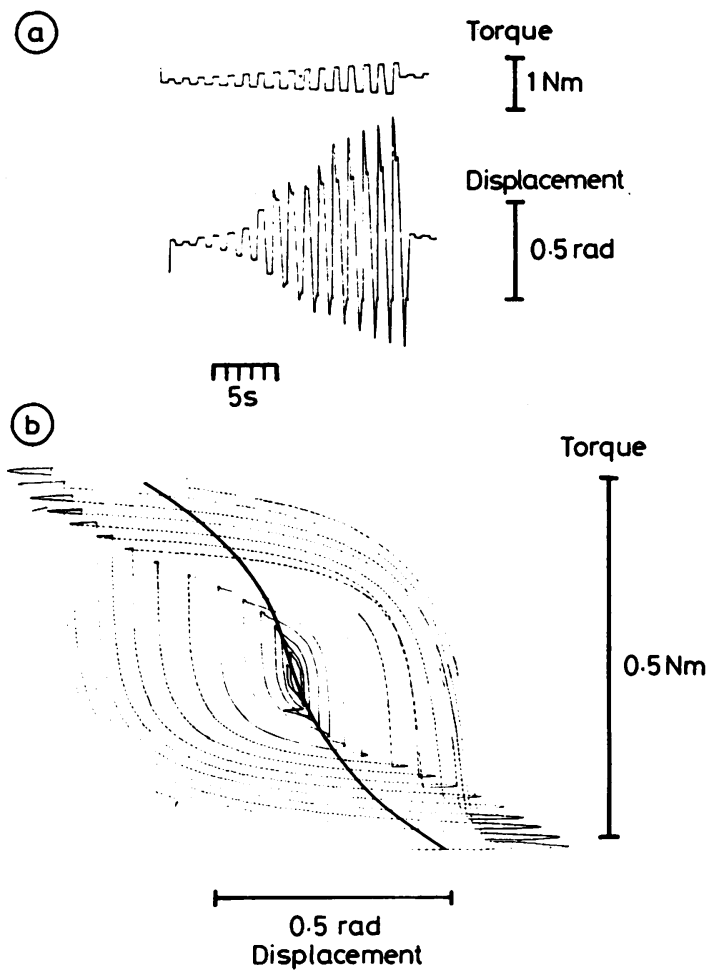

Fig 3 (A) The ramped rectangular driving torque and the resulting displacement of the wrist are shown. The size of the torque increases in linear steps but the displacement is very non-linear, being disproportionally small when the torque is low. In (B) the displacement has been plotted directly against torque using the $X-Y$ plotter. The locus of the stationary points is the composite compliance curve for the wrist. The disproportionate stiffness for small movements is again apparent. This figure also shows (heavy line) the composite compliance curve of the same subject following cooling of the forearm in water at $10^{\circ} \mathrm{C}$ for $30 \mathrm{~min}$. The stiffness for small forces is unaltered but becomes much greater as the forces increase. 


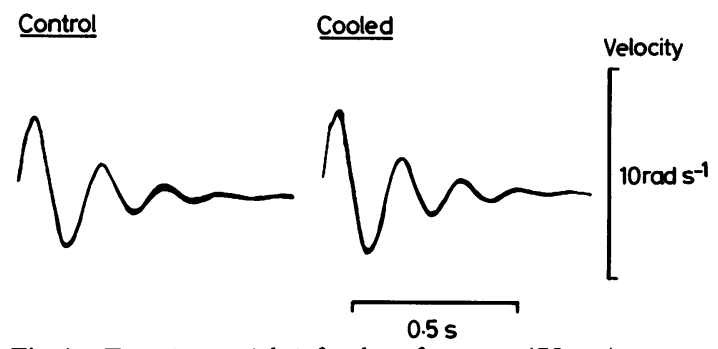

Fig 4 Transients. A brief pulse of current ( $75 \mathrm{~ms}$ ) through the motor applied a sharp tap to the hand. The velocity was recorded. The resulting transient oscillations of the wrist are shown. These are tracings from an oscilloscope of 6 superimposed taps. Cooling $\left(40 \mathrm{~min}\right.$ at $\left.6^{\circ} \mathrm{C}\right)$ does not change the frequency of these transients; thus the stiffness for small movements has not altered. Records of acceleration (obtained with the tremorograph) were similar.

applied. ${ }^{5}$ In fig $3 \mathrm{~B}$ the composite compliance curve for the same subject following cooling has been superimposed. In this case to preserve clarity only the curve has been drawn in and the points from which it was constructed are not shown. After cooling, the stiffness for small forces was unchanged, but the loosening that normally occurred with large forces was less, thus the displacement produced by the largest torques was relatively smaller. As in the previous experiments the effect of cooling has not been to increase the stiffness for small movements, but to reduce the ability of the muscles to become more compliant with larger movements. When larger forces were applied manually in an attempt to overcome the increased stiffness, discomfort in the stretched musculature was experienced. When the wrist and hand only were cooled by immersion in cold water for up to $\mathbf{4 0} \mathrm{min}$ there was no change in compliance.

In nine of these experiments the surface EMG from the flexor carpi ulnaris and extensor digitorum communis was recorded during the above investigations. In most subjects the movements did not cause observable discharges in the control periods or when cooled. The method was sensitive enough to detect a small voluntary movement of a finger. Some subjects showed shortening reactions during the control period, that is, the extensors became active as the hand was pushed into extension by the motor, and the flexors showed activity during flexion. After cooling these reactions became less prominent or disappeared, either because there was now less movement or because the sensitivity of the reflex arc that produced them may have been decreased by cooling. At no time was an increase of EMG produced by cooling; this was confirmed by integrating the EMG waveforms.

Most observations on the stiffness for small forces were made using the tremorograph (seven experiments on four male subjects, mean age 40 ). With this -instrument the hand was free to flex or extend in the horizontal plane. Small taps delivered manually or by a motor driven brush initiated a decrementing series of oscillatory transients at about $8 \mathrm{~Hz}$. Similar results were obtained using the Philips motor driven by a brief current pulse; the resultant velocity transients were recorded by the integral tachometer. Measurements made on anaesthetised and paralysed patients have shown that the stiffness producing these transients resides in the muscular system and is not dependent on neuromuscular control. ${ }^{5}$ In six experiments on three male subjects (mean age 46) we investigated transients before and after cooling the forearm. The recordings were not altered by cooling (fig 4) and the EMG was silent throughout. As the frequency of the transients was unchanged, the stiffness of the muscles for these small movements cannot have altered. This finding verifies the results described above. The decrement of the transients was similar in the cooled and control states indicating that the damping of the system had not changed.

\section{Voluntary movements and tracking}

Voluntary movements were studied in the course of the above experiments, but additional information came from tracking tasks (eight experiments, five subjects, one female, mean age 28) and from intrao muscular recording (two male subjects, mean age 47) During voluntary wrist movements activity could b recorded from several single units by a concentric needle electrode. As cooling progressed the number of units that could be seen decreased and the duration of the remainder was increased by about $30 \%$. These changes reversed on rewarming. These results may bre compared with those of Stalberg ${ }^{9}$ who noted a reduc=tion in the propagation velocity of single human muscle fibres in biceps brachialis with cooling to $23^{\circ} \mathrm{C}$. Below this temperature he could not elicit action potentials.
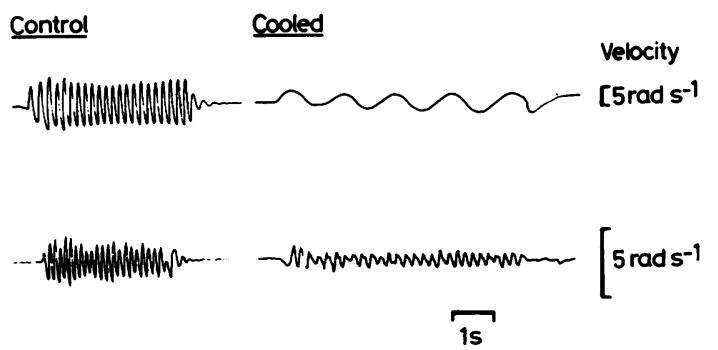

Fig 5 Voluntary movements. The subject was instructed to make reciprocating movements as fast as possible; he was able to keep the size of the movements nearly constant by monitoring the amplitude on a large screen oscilloscope. For large movements (top traces) cooling $\left(30 \mathrm{~min}\right.$ at $8^{\circ} \mathrm{C}$ ) reduced the rate from $6 \mathrm{~Hz}$ to less than $1 \mathrm{~Hz}$. Smaller movements (bottom trace) were much less affected. 

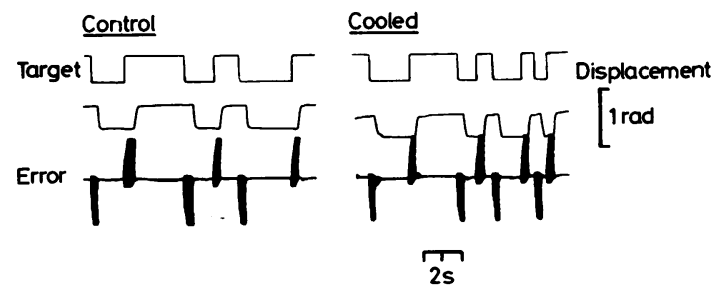

Fig 6 Tracking. The movement of the target and subject's spot are shown, and the difference between them (error). Cooling $\left(35 \min\right.$ at $\left.7^{\circ} \mathrm{C}\right)$ does not impair voluntary movements of this type.

Localised cooling causes striking changes in the control of voluntary movements. At normal temperatures, alternating flexion and extension movements of the wrist with an amplitude of about $0.5 \mathrm{rad}$ may be executed at a frequency of 5 or $6 \mathrm{~Hz}$. After cooling the forearm in water at $10^{\circ} \mathrm{C}$ for 30 min the maximum rate was less than $1.5 \mathrm{~Hz}$. With colder water a rate of $0.5 \mathrm{~Hz}$ became a struggle. When cooled, very small reciprocating movements could however still be made with reasonable facility (fig 5 ). Single movements of flexion or extension separated by a few seconds could be made almost as well as normal. We have called this selective inability to perform reciprocating movements "cold adiadokokinesia"10 When rapid reciprocating movements were attempted following cooling considerable voluntary effort was required for a laboured result, and there was often recruitment of motor activity in other parts of the body. Direct observation of the contours of the forearm revealed that with repeated movements reciprocal action was not occurring normally, for the flexors were still tense during the extension phase and viceversa. The EMG record following cooling showed a considerable increase in activity required to generate these alternating movements, and this was at time when fewer motor units remained operational.
These changes are reflected in records obtained during tracking tasks. There were two categories of target movement. In the first the subject was required to follow the abrupt jump of the spot from one side of the screen to the other. The jumps occurred at unpredictable intervals, but they were always separated by at least one second. This timing ensured that there was time for the muscles to relax before a movement in the opposite direction was required. After prolonged cooling at $9^{\circ} \mathrm{C}$ there was no deterioration in the performance of this task (fig 6). The second target movement was a sine wave which progressively increased in frequency. Above about $2 \mathrm{~Hz}$ true tracking is no longer possible because of the time delays which exist between eye and arm. At normal temperatures these time delays limit performance, for similar movements can be made at about three times this rate when not tracking. However, with practice, movements phase locked to the target were possible up to about $6 \mathrm{~Hz}$. After moderate cooling it became impossible to follow a spot at a frequency of more than $1 \mathrm{~Hz}$, with more prolonged cooling $0.5 \mathrm{~Hz}$ became marginal. The performance was not improved by long periods of practice or by limbering up.

It was noted that when the target speed was low (for example $0.1 \mathrm{~Hz}$ ) the performance at normal temperatures was imperfect as the motion generated was discontinuous. These imperfections were well seen in the acceleration record; they represented a physiological action tremor. After cooling these discontinuities were invariably greatly reduced (fig 7). In this sense, cooling might be said to improve tracking performance. We ascribe this improvement to the prolonged twitch time of the motor units so that the force input to the wrist became less spiky. A similar improvement in performance could be obtained at normal temperatures by attaching to the apparatus a vane immersed in treacle.

In four experiments (four male subjects, mean age

Control Cooled

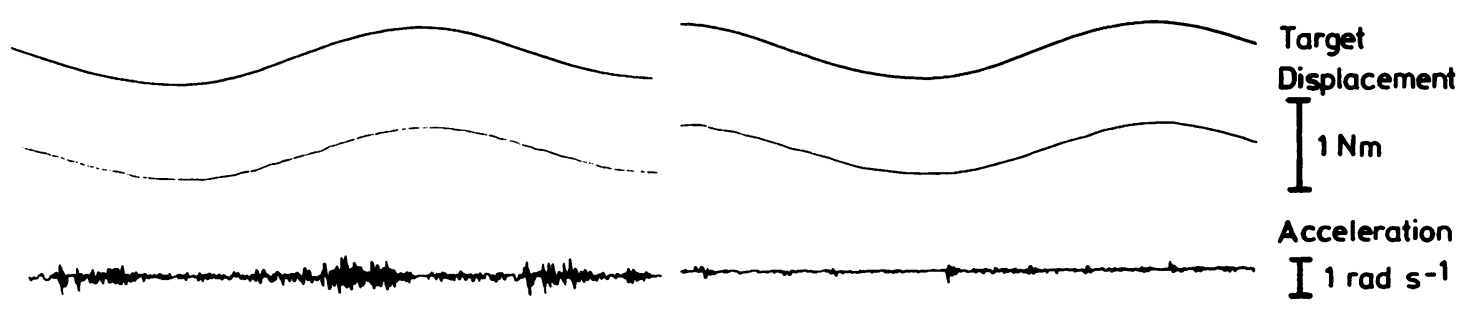

\section{$5 s$}

Fig 7 Tracking tremor. The discontinuities that are present when tracking (seen in the acceleration record) are much reduced by cooling. The degree of cooling is the same as for fig 6. 


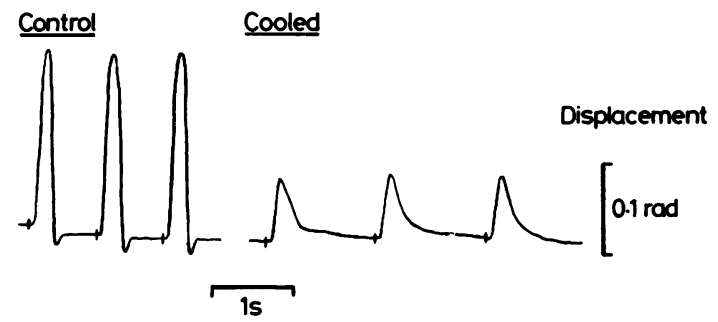

Fig 8 Electrically evoked twitches traced from a chart recorder. The twitches were isotonic against a constant low level force from the motor. Cooling $\left(40 \mathrm{~min}\right.$ at $\left.5^{\circ} \mathrm{C}\right)$ reduces the size of the twitch; the time to peak contraction is not much increased, but the relaxation time is prolonged, and the overshoots that are seen at normal temperatures are abolished.

42) the muscles of the forearm were stimulated by electric shocks before and after cooling. The hand was coupled to the printed motor which was supplied only with a steady current in order to stretch the extensor muscles being stimulated. The resulting isotonic twitch was recorded by the potentiometer. Isometric methods have traditionally been used to measure twitch times; the advantage of the isotonic method used here is that the conditions are the same as in the other experiments. A typical record is shown in fig 8 . The duration of the twitch was greatly increased by cooling, and the greatest prolongation was in the relaxation phase. In contrast, the latent period and contraction time were increased only slightly. There was also some decrease in the size of the twitch. The overshoots that were seen at normal temperatures were abolished.

\section{Discussion}

\section{Effectiveness of cooling}

We measured the temperature of the water in the bath, but not that of the forearm muscles. There must have been a gradient of temperature from the superficial to the deeper tissues and determinations at one site would have had little significance. Barcroft and Edholm ${ }^{11}$ sampled the deep temperature of the cooled forearm; with a bath temperature of $13^{\circ} \mathrm{C}$ the temperature in the deepest parts was $18^{\circ} \mathrm{C}$ after cooling for two hours. Clarke, et al ${ }^{12}$ found that the temperature of the forearm muscle at a depth of $1.5 \mathrm{~cm}$ was about $20^{\circ} \mathrm{C}$ after an immersion of $40 \mathrm{~min}$ in water at $1^{\circ} \mathrm{C}$. Accordingly, the deeper forearm muscles in our experiments will not have been nearly as cold as the circulating water.

\section{Passive properties}

The stiffness of the forearm can be greatly increased by localised cooling. Cooling of the wrist itself or the other forearm is without effect. The increase of stiffness is not accompanied by an increased EMG; consequently we conclude that the changes produced by cooling are a direct effect on the muscle tissue itself. Muscular rigidity has been described as a symptom of accidental hypothermia ${ }^{113}$ and has sometimes been attributed to overactivity of spinal reflexes.

In our experiments changes in stiffness can be observed after about $15 \mathrm{~min}$ in water at $8^{\circ} \mathrm{C}$ and are well developed after $30 \mathrm{~min}$. The change is not a simple increase in spring stiffness, but is produced by a non-neurally mediated change in the complex thixotropic properties of the muscles. Thus, for small forces, the stiffness of the limb is not increased by cooling; this is shown by the lack of change in the transients induced by a tap, and the unchanged response to small sinusoidal and rectangular torques. When larger forces are applied the situation is different. The amount of movement is now much less than it was before cooling and the ability of a perturbation to loosen the system is lost. The stiffness of a tissue is inversely related to the elongation that results from an applied force. In a linear (Hookian) system the same value of stiffness would be obtained at all force levels. The wrist, however, is very nonlinear, being considerably stiffer for small movements It has been known for some time that relaxed anima? muscle is stiffer for small rather than large move $\frac{\mathrm{O}}{\mathrm{D}}$ ments; ${ }^{14-16}$ the "preliminary rigidity" of Denny Brown. ${ }^{14}$ Our hypothesis ${ }^{5}$ is that when stationary, of with minimal agitation the musculature undergoes of transition from a state resembling a sol to a state resembling a gel. With movements of greater size the system reverts to the sol state and becomes more com $=$ pliant. Thus the muscles will always be stiff about their resting position. Thixotropic effects are due to a sol-gel transformation. Weak bonds (between the thick and thin filaments) are broken by agitation and, once disrupted, take time to reform. The stiffness is therefore dependent on the previous history of movement. It might be expected that cooling would reduce the disruptive effects of Brownian motion on the weak physico-chemical bonds involved and favour the gel rather than the sol state. A similar mechanism has been observed by Edwards et al. in active muscle; ${ }^{3}$ warming the human quadriceps muscle was shown to increase the metabolic cost of maintaining a contraction and cooling decreased the relaxation rate following a twitch. These authors have suggested that at low temperatures the bonding of actin and myosin becomes greater. We believe that it is the inability of the cooled muscles to loosen up which underlies these results and that they can be best explained on the basis of cold induced changes in muscle thixotropy. Thus, the ability to "limber up" is lost when the muscles are cooled. 
The muscle relaxing effect of a soak in a hot bath or other forms of heat treatment may be explicable on this basis. However, some physiotherapists use topical cryotherapy in an attempt to reduce the muscular hypertonicity of spasticity. ${ }^{17}$ The results of local cooling of spastic muscles may be different. This effect may be due to changes in reflex control. It has been shown that primary and secondary muscle spindles and tendon organs fire at reduced rates when cooled and subjected to tensions greater than that required to sustain a steady discharge. ${ }^{18}$

\section{Active properties}

It has been long established that cooling of mammalian skeletal muscle changes its twitch time. Tuttle $^{19}$ working with the cooled human calf muscles under isotonic conditions, showed that the latency and contraction time were slightly increased, but the relaxation time was more than doubled. DK Hill ${ }^{16}$ found that the isometric twitch of the rat soleus became remarkably prolonged at low temperatures. The retardation with cooling was principally in the relaxation phase. Cooling the forearm muscles in our experiments decreased the twitch size and greatly increased the time for relaxation. The inability to relax promptly and the decrease in twitch size can explain the difficulty in making rapid reciprocating movements. AV Hill ${ }^{20}$ suggested that an athlete would run faster if his muscles were warmed; certainly rapid alternating movements cannot be made if the muscles are cold. There is a report of a swimmer who was unable to move and had muscular rigidity after $33 \mathrm{~min}$ in water at $16^{\circ} \mathrm{C} .{ }^{21}$ Under conditions where forces act against muscles that are imperfectly relaxed greater than normal stresses may arise; this may predispose to injury of muscles or tendons in cold conditions.

\section{Metabolic effects}

Muscle tone has long been associated with the production and regulation of body heat. We have elsewhere considered resting muscle tone and have found no evidence that nervous discharges are responsible. ${ }^{5}$ As resting muscle tone results from a passive process only trivial amounts of heat may be expected to be generated. Working with the relaxed human forearm, Holling 22 estimated that the oxygen consumption was as low as $0.07 \mathrm{ml} / \mathrm{g} / \mathrm{h}$. If this figure can be applied to all the muscles of the body it represents only about $14 \%$ of the BMR.

Since the last century there have been reports that the metabolic rate increases as the body is cooled and before shivering commences. ${ }^{23}$ This "pre-shivering hypertonia" has been attributed to a neurally mediated increased in muscle tone. There appears to have been little interest in this subject since satisfactory EMG recordings became possible. We have traced only a note ${ }^{24}$ and a short paper. ${ }^{25}$ The present results suggest that at least in part any increase in muscle tone that occurs following cooling may be a direct effect on the muscle tissues; this is unlikely to have significant metabolic consequences. Some workers now believe that even in adults cold induced thermogenesis may be due to brown fat. The phenomenon has been shown to persist in patients following neuromuscular blockade. ${ }^{26}$ In infants, ${ }^{27}$ and no doubt in some adults, a cold environment may induce restlessness which may raise the metabolic rate by intermittent muscular activity.

The support of the Royal Society and the Medical Research Council is gratefully acknowledged.

\section{References}

${ }^{1}$ Laufman $\mathrm{H}$. Profound accidental hypothermia. JAMA 1951;147:1201-12

${ }^{2}$ Bergh U, Ekblom B. Influence of muscle temperature on maximal muscle strength and power output in human skeletal muscles. Acta physiol Scand 1976;107:33-37.

${ }^{3}$ Edwards RHT, Harris RC, Hultman E, Kaijser L, Koh D, Nordensjo L-O. Effect of temperature on muscle energy, metabolism and endurance during successive isotonic contractions, sustained to fatigue, of the quadriceps muscle in man. $J$ Physiol (Lond) 1972;222:335-52.

${ }^{4}$ Provins KA, Clarke RS. The effect of cold on manual performance. J Occup Med 1968;2:169-76.

${ }^{5}$ Lakie M, Walsh EG, Wright GW. Resonance at the wrist demonstrated by the use of a torque motor, an instrumental analysis of muscle tone in man. $J$ Physiol (Lond) 1984;353:265-85.

${ }^{6}$ Marsden CE, Merton PA, Morton HB. Servo action in the human thumb. J Physiol (Lond) 1976;257:1-44.

${ }^{7}$ Walsh EG, Wright GW. A hanging-hand tremorograph. $J$ Physiol (Lond) 1982;329:1-2P.

${ }^{8}$ Lakie M, Scott DB, Walsh EG, Wright GW. Resonance at the wrist in anaesthetised subjects. J Physiol (Lond) 1983;338:32P.

${ }^{9}$ Stalberg E. Propagation velocity in human muscle fibres in situ. Acta Physiol Scand 1966;70:287-399.

${ }^{10}$ Lakie M, Walsh EG. Cold adiadokokinesia. J Physiol (Lond) 1982;328:41-42P.

${ }^{11}$ Barcroft $\mathrm{H}$, Edholm OG. The effect of temperature on blood flow and deep temperature in the human forearm. J Physiol (Lond) 1943;102:5-20.

${ }^{12}$ Clarke RSJ, Helon RF, Lind AR. Vascular reactions of the human forearm to cold. Clin Sci 1958;17:165-79.

${ }^{13}$ Simpson JA. On the muscular rigidity and hyperreflexia due to hypothermia in man with observations on-the accommodation of peripheral nerve. J Neurol Neurosurg Psychiatry 1955;18:191-5.

${ }^{14}$ Denny-Brown DE. On the nature of postural reflexes. Proc R Soc (Biol) 1929;104:252-301.

${ }^{15}$ Nichols TR, Houk JC. Improvement in linearity and regulation of stiffness that results from actions of the 
stretch reflex. $J$ Neurophysiol 1976;39.1:119-42.

${ }^{16} \mathrm{Hill}$ DK. Resting tension and the form of the twitch of rat skeletal muscle at low temperature. J Physiol (Lond) 1972;221:161-71.

${ }^{17}$ Knutson E. Topical cryotherapy in spasticity. Scand $J$ Rehab Med 1970;2:159-63.

${ }^{18}$ Eldred E, Lindsley DF, Buchwald JS. The effect of cooling on mammalian muscle spindles. Exp Neurol 1960;2:144-57.

${ }^{19}$ Tuttle WW. The effects of decreased temperature on the activity of intact muscle. $J$ Lab Clin Med 1941;26:1913-9.

${ }^{20}$ Hill AV. Trails and Trials in Physiology. 1965 London: Arnold.

${ }^{21}$ Pugh LGC, Edholm OG. The physiology of channel swimmers. Lancet 1955;2:761-8.

${ }^{22}$ Holling HE. Observations on the oxygen content of venous blood from the arm vein and the oxygen con- sumption of resting human muscle. Clin Sci 1939;4:103-11.

${ }^{23}$ Cannon WB, Querido A, Britton SW, Bright EM. Studies in the conditions of activity in endocrine glands XXI The role of adrenal secretion in the chemical control of body temperature. Am J Physiol 1927;79:466-507.

${ }^{24}$ Burton AC, Bronk DW. The motor mechanism of shivering and of thermal muscle tone. Am J Physiol 1937;119:284.

${ }^{25}$ Petajan JH, Williams DD. Behaviour of single motor units during pre-shivering tone and shivering tremor. $A m J$ Phys Med 1972;51:16-22.

${ }^{26}$ Jessen K, Rabol A, Winkler K. Total body and splanchnic thermogenesis in curarised man during a short exposure to cold. Acta Anaesthesiol Scand 1980;24:339-44.

${ }^{27}$ Hey EN. The relation between environmental temperature and oxygen consumption in the new-born baby. J Physiol (Lond) 1969;200:589-603. 